\title{
GERMANIUM PRODUCTION IN ASYMPTOTIC GIANT BRANCH STARS: IMPLICATIONS FOR OBSERVATIONS OF PLANETARY NEBULAE
}

\author{
AMANDA I. KARAKAS ${ }^{1,2}$ \\ Origins Institute and Department of Physics and Astronomy, McMaster University, Hamilton, ON, Canada; karakas@physics.mcmaster.ca \\ MARIA LUGARO ${ }^{2}$ \\ Sterrenkundig Institute, University of Utrecht, Utrecht, Netherlands; M.Lugaro@phys.uu.nl \\ AND \\ Roberto Gallino ${ }^{2}$ \\ Dipartimento di Fisica Generale, Universitá di Torino, Torino, Italy; gallino@ph.unito.it \\ Received 2006 August 22; accepted 2007 January 11; published 2007 February 2
}

\begin{abstract}
Observations of planetary nebulae (PNe) in the work of Sterling, Dinerstein, \& Bowers have revealed abundances in the neutron-capture element germanium $(\mathrm{Ge})$ from solar to factors of 3-10 above solar. The enhanced $\mathrm{Ge}$ is an indication that the slow neutron-capture process (the s-process) operated in the parent star during the thermally pulsing asymptotic giant branch (TP-AGB) phase. We compute the detailed nucleosynthesis of a series of AGB models to estimate the surface enrichment of Ge near the end of the AGB. A partial mixing zone of constant mass is included at the deepest extent of each dredge-up episode, resulting in the formation of a ${ }^{13} \mathrm{C}$ pocket in the top approximately one-tenth of the He-rich intershell. All of the models show surface increases of $[\mathrm{Ge} / \mathrm{Fe}] \lesssim 0.5$, except the $2.5 M_{\odot}, Z=0.004$ case, which produced a factor of 6 enhancement of Ge. Near the tip of the TP-AGB, a couple of extra thermal pulses (TPs) could occur to account for the composition of the most Ge-enriched PNe. Uncertainties in the theoretical modeling of AGB stellar evolution might account for larger Ge enhancements than we predict here. Alternatively, a possible solution could be provided by the occurrence of a late TP during the post-AGB phase. Difficulties related to spectroscopic abundance estimates also need to be taken into consideration. Further study is required to better assess how the model uncertainties affect the predictions and, consequently, if a late TP should be invoked.
\end{abstract}

Subject headings: nuclear reactions, nucleosynthesis, abundances — planetary nebulae: general — stars: AGB and post-AGB

Online material: color figure

\section{INTRODUCTION}

After the TP-AGB phase is terminated, low- to intermediatemass stars (with initial masses $\sim 0.8-8 M_{\odot}$ ) evolve through the PNe phase before ending their lives as white dwarfs (see the recent review by Herwig 2005 and references therein). The whole envelope is lost because of the blowing of low-velocity stellar winds during the AGB phase. The gaseous nebula is the remnant of the deep convective envelope that once surrounded the core, which is now exposed as the central star (CS) of the illuminated nebula. Thus, the abundances of the nebula should reveal information about the chemical processing that took place during the AGB and, more precisely, information about the last TPs. The spectra of PNe are usually a composite, a mixture from the nebula and the illuminating central star.

Accurate abundances from PNe for helium, C, N, O, Ne, S, Cl, and Ar are available (Kaler 1978; Henry 1989; Dopita et al. 1997; Stanghellini et al. 2000). These abundances can be used as a powerful tool to constrain models of AGB stars (Karakas \& Lattanzio 2003), in particular, the amount of mixing that occurs during third dredge-up (TDU) episodes following a TP. The discovery in PNe of lines of the light neutron-capture element Ge by Sterling et al. (2002) provides not only constraints on the amount of dredge-up but also on the operation of the $s$-process occurring in AGB stars. Despite the solar Ge

\footnotetext{
${ }^{1}$ Current address: Australian National University, Research School of Astronomy and Astrophysics, Mount Stromlo Observatory, Canberra, ACT, Australia.

${ }^{2}$ Centre for Stellar and Planetary Astrophysics, Monash University, Clayton, VIC, Australia.
}

abundance being dominated by massive star nucleosynthesis, with AGB stars only contributing 6\% of solar Ge (Arlandini et al. 1999; Busso et al. 2001), this element can be produced by the $s$-process in sufficient quantities to be observed in PNe.

In this Letter we seek to address the question of whether detailed models of AGB stars can reproduce the observed amount of $\mathrm{Ge}$ in PNe. This study is timely given the increasing number of detections of heavy elements in $\mathrm{PNe}$, and indeed this is the first study dedicated to comparing these abundances with AGB nucleosynthesis results. In a follow-up paper we will expand on this work to cover all elements up to the first $s$ peak.

\section{THE NUMERICAL METHOD}

The numerical method we use has been previously described in detail (Lugaro et al. 2004; Karakas et al. 2006). Here we summarize the main points relevant for this study. We first compute the structure and then perform detailed nucleosynthesis calculations. The reaction network in the post-processing algorithm has been expanded from 74 species to 156 , to include all stable isotopes up to ${ }^{75} \mathrm{As}$. We include all $\beta$-decays up to arsenic, and all $p-, \alpha$-, and $n$-capture reactions on all species in the network, which results in a total of 1260 reaction rates.

To account for neutron captures on elements heavier than arsenic, we use a "double neutron sink" description (Herwig et al. 2003) and include two artificial species that are linked by the following reactions: ${ }^{75} \mathrm{As}(n, \gamma)^{76} \mathrm{~g}$ and ${ }^{76} \mathrm{~g}(n, L)^{76} \mathrm{~g}$, where ${ }^{76} \mathrm{~g}$ replaces ${ }^{76} \mathrm{As}$ and has an initial abundance equal to the sum 
TABLE 1

Details of The Stellar Models

\begin{tabular}{lcccccccc}
\hline \hline Mass & $Z$ & TPs & $T_{\mathrm{He}}^{\max }$ & Mass $_{\text {dred }}$ & HBB? & $\mathrm{C} / \mathrm{O}$ & ${ }^{12} \mathrm{C} /{ }^{13} \mathrm{C}$ & $M_{\text {env }}$ \\
\hline 3.0 & 0.02 & 26 & 302 & $8.11(-2)$ & No & 1.40 & 118.0 & 0.676 \\
5.0 & 0.02 & 24 & 352 & $5.03(-2)$ & Yes & 0.77 & 7.840 & 1.500 \\
6.5 & 0.02 & 40 & 368 & $4.70(-2)$ & Yes & 0.40 & 11.60 & 1.507 \\
3.0 & 0.012 & 22 & 307 & $9.09(-2)$ & No & 2.79 & 185.0 & 0.805 \\
6.5 & 0.012 & 51 & 369 & $6.48(-2)$ & Yes & 0.76 & 10.40 & 1.389 \\
1.9 & 0.008 & 19 & 278 & $1.70(-2)$ & No & 2.08 & 143.0 & 0.221 \\
2.5 & 0.004 & 30 & 308 & $8.17(-1)$ & No & 14.7 & 1416 & 0.685 \\
\hline
\end{tabular}

of solar abundances from $\mathrm{Se}$ to $\mathrm{Bi}$. The second artificial particle, $L$, is equivalent to counting the number of neutrons captured beyond arsenic. The ratio $\left(L /^{76} \mathrm{~g}\right)$ is a description of the neutrons captured per seed nuclei and could, in principle, be related to the $s$-process distribution.

Most of the 1260 reaction rates are from the 1991 updated REACLIB Data Tables (Thielemann et al. 1986). Many of the $p-, \alpha$, and $n$-capture reaction rates have been updated by $\mathrm{Lu}-$ garo et al. (2004). We use the NACRE (Angulo et al. 1999) rates for the $\mathrm{NeNa}$ and $\mathrm{MgAl}$ chains, and rates from Karakas et al. (2006) for the ${ }^{22} \mathrm{Ne}+\alpha$ reactions. The cross section of the ${ }^{76} \mathrm{~g}(n, L)^{76} \mathrm{~g}$ reaction is a composite calculated using heavyelement distributions produced by the $s$-process in low-mass AGB stars from the models of Gallino et al. (1998). Test simulations show that using a different cross section for ${ }^{76} \mathrm{~g}$ does not significantly affect the elemental abundance of Ge (or any other species) in a $3 M_{\odot}, Z=0.012$ model or a $2.5 M_{\odot}$, $Z=0.004$ model.

\section{THE STELLAR MODELS}

We compute stellar models covering a range of initial masses and metallicities, which are listed in the first two columns of Table 1. The metallicities were chosen to reflect the composition of the progenitor AGB stars that were estimated to range from $0.3 Z_{\odot}$ to $Z_{\odot}$ (Sterling \& Dinerstein 2005). The $Z=0.012$ stellar models were computed with the revised solar elemental abundances from Asplund et al. (2005) for comparison to the $Z=0.02$ models with abundances from Anders \& Grevesse (1989). Scaled solar abundances were assumed for the $Z=$ 0.008 and 0.004 models. We use the mass loss from Vassiliadis \& Wood (1993) on the AGB.

In Table 1 we include the number of TPs, the maximum temperature in the He shell, the total mass dredged into the envelope during the AGB, the final surface $\mathrm{C} / \mathrm{O}$ and ${ }^{12} \mathrm{C} /{ }^{13} \mathrm{C}$ ratios, and the envelope mass at the last computed time step. All masses are in solar units, and the temperature is in units of $10^{6} \mathrm{~K}$. From Table 1 we see that the 3 and $6.5 M_{\odot}, Z=$ 0.012 models were similar to their $Z=0.02$ counterparts. The low-mass AGB models became carbon stars, whereas those models with hot bottom burning (HBB) retained an O-rich atmospheric composition (5 and $6.5 M_{\odot}$ ). There is evidence (e.g., Abia \& Isern 1997) that some extra mixing takes place in $\sim 1-3 M_{\odot}$ AGB stars, resulting in lower observed ${ }^{12} \mathrm{C} /{ }^{13} \mathrm{C}$ and $\mathrm{C} / \mathrm{O}$ ratios than predicted by models such as ours.

The results presented in this study are subject to many model uncertainties, including convection and mass loss, that affect both the structure and the nucleosynthesis. We refer to Goriely \& Mowlavi (2000), Herwig (2005), Ventura \& D'Antona (2005), and Straniero et al. (2006) for detailed discussions on this topic.

\section{THE INCLUSION OF A PARTIAL MIXING ZONE}

The inclusion of a partial mixing zone (PMZ) at the deepest extent of dredge-up will mix protons from the envelope into
TABLE 2

[Ge/Fe] And [Ga/Fe] Ratios after the Final Computed TP

\begin{tabular}{cccccc}
\hline \hline Mass & \multicolumn{1}{c}{$Z$} & $M_{\text {csh }}$ & PMZ & {$[\mathrm{Ge} / \mathrm{Fe}]$} & {$[\mathrm{Ga} / \mathrm{Fe}]$} \\
\hline $3.0 \ldots \ldots$ & 0.02 & $1.6(-2)$ & $2.0(-3)$ & 0.474 & 0.453 \\
$5.0 \ldots \ldots$ & 0.02 & $4.9(-3)$ & $1.0(-4)$ & 0.112 & 0.111 \\
$6.5 \ldots \ldots$ & 0.02 & $2.3(-3)$ & None & 0.191 & 0.212 \\
$3.0 \ldots \ldots$ & 0.012 & $1.6(-2)$ & $1.0(-3)$ & 0.386 & 0.482 \\
& & & $2.0(-3)$ & 0.557 & 0.678 \\
& & & $\ldots$ & $0.557^{\mathrm{a}}$ & $0.677^{\mathrm{a}}$ \\
$6.5 \ldots \ldots$ & 0.012 & $2.5(-3)$ & None & 0.186 & 0.304 \\
$1.9 \ldots \ldots$ & 0.008 & $2.0(-2)$ & $2.0(-3)$ & 0.252 & 0.333 \\
& & & $3.0(-3)$ & 0.339 & 0.439 \\
$2.5 \ldots \ldots$ & 0.004 & $1.7(-2)$ & $2.0(-3)$ & 0.777 & 0.894
\end{tabular}

Note. - Models with no PMZ have $[\mathrm{Ge} / \mathrm{Fe}] \approx 0$ for all cases but the $6.5 M_{\odot}$ models. All masses are in solar masses.

${ }^{a}$ These values are the $[\mathrm{Ge} / \mathrm{S}]$ and $[\mathrm{Ga} / \mathrm{S}]$ ratios, computed using sulfur as the reference element.

the He intershell, producing a ${ }^{13} \mathrm{C}$ pocket. In the $\mathrm{PMZ}$, neutrons are liberated during the interpulse period by the reaction ${ }^{13} \mathrm{C}(\alpha$, $n)^{16} \mathrm{O}$, and they are captured by $\mathrm{Fe}$-seed nuclei to produce heavy elements. Observational and theoretical evidence suggests that this is the dominant neutron source in low-mass AGB stars (Smith et al. 1987; Gallino et al. 1998). The details of how the pocket forms and its extent in mass in the $\mathrm{He}$ intershell are still unknown, although gravity waves (Denissenkov \& Tout 2003), convective overshoot (Herwig 2000), induced overshoot during the TDU episodes (Cristallo et al. 2004; Straniero et al. 2006), and rotationally induced mixing (Langer et al. 1999; Herwig et al. 2003) have been suggested.

The ${ }^{22} \mathrm{Ne}(\alpha, n)^{25} \mathrm{Mg}$ reaction produces a brief strong burst of neutrons in the convective pocket during a TP when the temperature exceeds $\sim 300 \times 10^{6} \mathrm{~K}$. This is probably the dominant neutron source in massive AGB stars, where the mass of the $\mathrm{He}$ shell is smaller by about an order of magnitude compared to lower mass stars, reducing the importance of the ${ }^{13} \mathrm{C}$ pocket.

As done in previous nucleosynthesis studies (Gallino et al. 1998; Goriely \& Mowlavi 2000; Lugaro et al. 2004), we artificially include a PMZ of constant mass. In Table 2 we show the maximum mass of the pulse-driven convective shell, $M_{\mathrm{csh}}$, at the last TP along with the PMZ masses used. Note that $M_{\text {csh }}$ is approximately equal to the He-intershell mass during the TP. The PMZ mass is estimated to be between $\sim 5 \%$ and $15 \%$ of the mass of the He-rich intershell, and we used PMZs of $1 \times 10^{-3}$ and $2 \times 10^{-3} M_{\odot}$ for the $3 M_{\odot}$ models. We chose a proton profile in which the number of protons decreases exponentially with the mass depth below the base of the convective envelope in the same way as described in Lugaro et al. (2004). We also computed models with no PMZ for all masses, in order to single out the effect of the ${ }^{22} \mathrm{Ne}$ source.

\section{RESULTS}

In Table 2 we show the surface $[\mathrm{Ge} / \mathrm{Fe}]$ and $[\mathrm{Ga} / \mathrm{Fe}]$ ratios after the last computed TP for each stellar model, where we use Fe as the reference element, which is justified because the surface abundance is predicted by the models to be unaltered by AGB nucleosynthesis. ${ }^{3}$ We also provide the surface $[\mathrm{Ge} / \mathrm{S}]$ and $[\mathrm{Ge} / \mathrm{S}]$ ratios for the $3 M_{\odot}, Z=0.012$ model (with a PMZ $=2 \times 10^{-3} M_{\odot}$ ) for comparison. There is no difference between these values and those computed using Fe as the reference element. Sterling \& Dinerstein (2003) tentatively detected Ga in one PN (SwSt 1), with the derived $[\mathrm{Ga} / \mathrm{S}]=1.64-2.87$, depending on the level of depletion into dust. Table 2 shows that the largest predicted en-

${ }^{3}$ Sulfur is similarly not altered. 

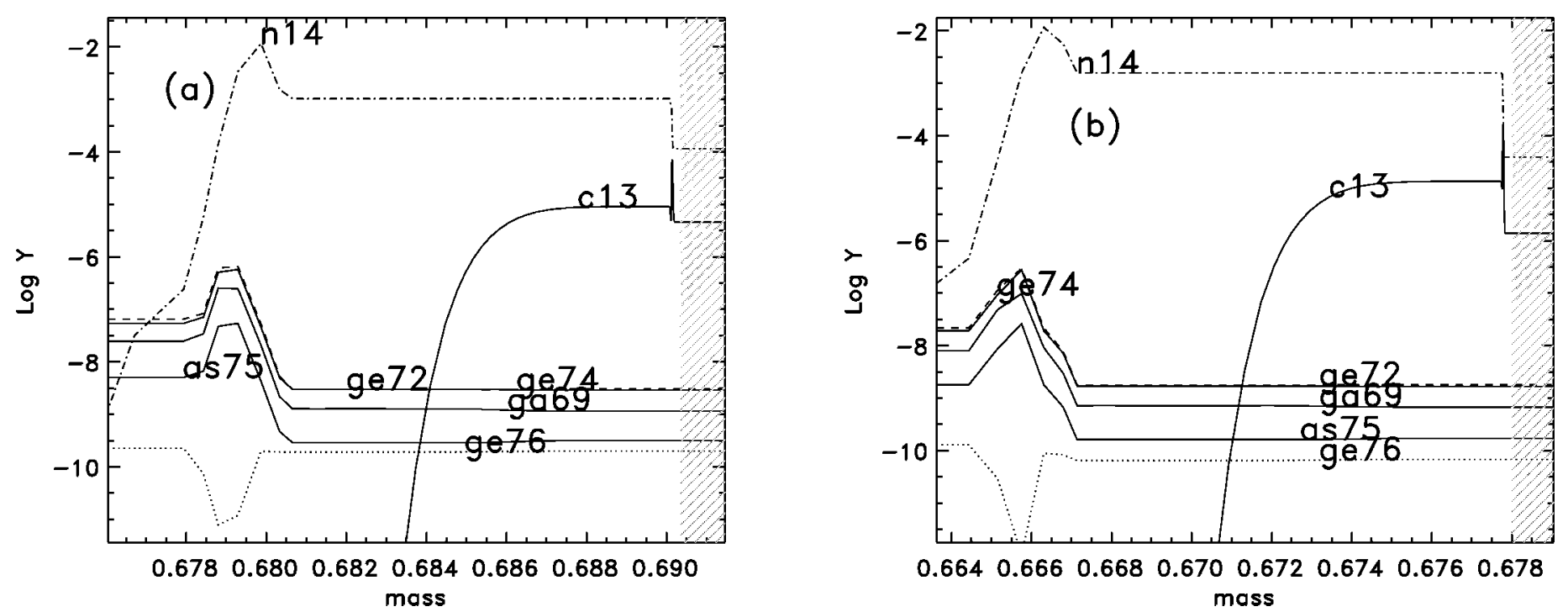

FIG. 1.-Composition profile showing the intershell abundances (in $\log Y$, where mass fraction $X=Y A$ ) just before the last computed TP. The shaded region is the inner edge of the convective envelope, and a PMZ of $0.002 M_{\odot}$ was used in both models. We show abundances for selected isotopes from (a) the $3 M_{\odot}$, $Z=0.012$ model, in which the Ge intershell abundance after the last TP is 40 times solar, and $(b)$ the $2.5 M_{\odot}, Z=0.004$ model, in which the Ge intershell abundance is 63 times solar. The intershell abundances are typically diluted by 1 order of magnitude at the surface by the last TDU episode. [See the electronic edition of the Journal for a color version of this figure.]

hancements in terms of $[\mathrm{Ge} / \mathrm{Fe}]$ were $₫ 0.56$ (or a factor of $\sim 3.6$ ) for all cases but the $2.5 M_{\odot}, Z=0.004$ model, in which $[\mathrm{Ge} / \mathrm{Fe}]=0.77$. Models with no ${ }^{13} \mathrm{C}$ pocket produced very little $\mathrm{Ge}$ and $\mathrm{Ga}$, except in the case of the $6.5 M_{\odot}$ solar metallicity models. For these massive AGB models, neutrons are released by efficient activation of the ${ }^{22} \mathrm{Ne}$ neutron source during TPs, and the increase of $\mathrm{Ge}$ at the tip of the TP-AGB is a factor of $\sim 1.5$ above solar. In comparison, the $5 M_{\odot}, Z=0.02$ model produced little Ge with $([\mathrm{Ge} / \mathrm{Fe}]=0.112)$ or without $(0.04)$ a PMZ.

The $2.5 M_{\odot}, Z=0.004$ model produced the most Ge, partly because efficient TDU results in a large amount of matter dredged from the core into the envelope (see Table 1). Another reason is that the efficiency of the ${ }^{13} \mathrm{C}(\alpha, n){ }^{16} \mathrm{O}$ neutron source does not depend on the initial $Z$, and that means more neutrons per Fe-seed nuclei are produced at lower metallicity. Table 1 from Goriely \& Mowlavi (2000) also shows more Ge in the dredged-up matter with decreasing $Z$. Figures 18 and 20 in Gallino et al. (1998) show the $s$-process enhancement factors in the intershell material cumulatively dredged into the envelope in $2 M_{\odot}$ models with $Z=0.01$ and $Z=0.0005$, respectively. The lower $Z$ model clearly shows an increase in the production of the Ge isotopes, with atomic mass $\approx 70$.

Our results compare favorably with the $3 M_{\odot}, Z=0.02$ model computed with the FRANEC evolutionary code (Straniero et al. 1997) by Gallino et al. (1998) with their standard ${ }^{13} \mathrm{C}$ pocket (hereafter the Torino models); they find that the final $[\mathrm{Ge} / \mathrm{Fe}],[\mathrm{Ga} / \mathrm{Fe}],[\mathrm{Zr} / \mathrm{Fe}]$, and $[\mathrm{Ba} / \mathrm{Fe}]$ equal $0.46,0.43$, 1.03 , and 0.93 , respectively. The $1.5 M_{\odot}, Z=0.02$ produced values equal $0.28,0.29,0.75$, and 0.65 , respectively. The Torino $5 M_{\odot}, Z=0.02$ model produced a final $[\mathrm{Ge} / \mathrm{Fe}]=0.57$, higher than our equivalent model, owing to the choice of the Reimers (1975) mass loss (with $\eta=10$ ), which results in more TPs (48) compared to our computation (24). Massive AGB stars have been suggested as the progenitors of Type I bipolar PNe owing to their high $\mathrm{He}$ and N/O abundances (Stanghellini et al. 2006) as well as their kinematics (Corradi \& Schwarz 1995). Sterling \& Dinerstein (2005) and Sterling (2006) find a correlation between PNe morphology and s-process overabundances, where elliptical PNe are more enriched than bipolar PNe, which show little or no enhancement. These ob- servations suggest that Reimers-type mass loss is inadequate for modeling massive AGB stars and that formulae with a superwind (such as Vassiliadis \& Wood 1993) are favored.

None of the AGB models lost all of their envelopes before the computation finished. That is, all of the models were near the tip of the AGB but had not proceeded to the post-AGB phase. Some of these models could, in principle, experience further TPs and TDUs, and we refer to these as extra TPs. The amount of Ge expected after the $i$ th TP can be estimated according to $X^{i}=$ $\left(M_{\mathrm{env}}^{i-1} X^{i-1}+\Delta M_{\mathrm{Ge}}\right) / M_{\mathrm{env}}^{i}$, where $\Delta M_{\mathrm{Ge}}=\lambda \Delta M_{\text {intershell }}$ is the mass of Ge dredged into the envelope, $\lambda$ is the TDU efficiency, and $\Delta M_{\text {intershell }}$ is the mass of Ge in the intershell. In Figure 1 we show the intershell abundances for selected Ge isotopes for two models. A Ge pocket can be seen in both cases, and although this will be diluted by 1 order of magnitude by intershell convection, the post-pulse abundances are still enhanced compared to solar.

Using the $3 M_{\odot}, Z=0.012$ model with a PMZ of $2 \times$ $10^{-3} M_{\odot}$ as an example, we estimate that the next TP would take place when $M_{\mathrm{env}} \approx 0.7 M_{\odot}$. The mass of the He intershell at the last TP is $0.016 M_{\odot}$, and assuming the same TDU efficiency as in the previous TP, $\lambda=0.835$. At the last computed time step, the Ge envelope and intershell compositions are $9.64 \times 10^{-9}$ and $1.068 \times 10^{-7}$ (in mass fractions), respectively, from which we estimate that after the next TP, $[\mathrm{Ge} / \mathrm{Fe}]=0.64$. If we assume a mass-loss rate of $10^{-5} M_{\odot} \mathrm{yr}^{-1}$ (typical near the end of the AGB) multiplied by the interpulse period of 54,000 years, then $\sim 0.54 M_{\odot}$ is lost per interpulse. ${ }^{4}$ From this we estimate that the final TP would occur when $M_{\text {env }} \sim 0.15 M_{\odot}$. We can speculate that if a TDU episode occurs with the same efficiency as before, and using the envelope and intershell $\mathrm{Ge}$ abundances from the previous estimate $\left(1.17 \times 10^{-8}\right)$, we get $[\mathrm{Ge} / \mathrm{Fe}]=0.90$ (or $\mathrm{Ge}=2.14 \times 10^{-8}$ in mass fractions), at the tip of the AGB, close to the value required to match the composition of the most Geenriched PNe. However, caution is required because this value is very likely to be an overestimate, owing to the evidence of a decreasing TDU efficiency with a decreasing envelope mass (Straniero et al. 1997). Variations in AGB mass-loss rates and

\footnotetext{
${ }^{4}$ The same mass model computed with an interpulse period of 25,000 years near the end of the AGB would allow for two to three more TPs.
} 
model-dependent parameters such as the interpulse period will also effect this estimate.

Increasing the size of the PMZ in the models produces more $\mathrm{Ge}$, but if we increase the size of the pocket too much, we run into the problem that the PMZ accounts for a large percentage of the intershell region. This is contrary to simulations that suggest that the ${ }^{13} \mathrm{C}$ pocket should be, at most, $\sim 10 \%$ of the intershell (Denissenkov \& Tout 2003; Lugaro et al. 2003; Herwig 2005).

\section{DISCUSSION AND CONCLUSIONS}

The results presented in this Letter show that AGB models can explain the spread of Ge abundance in PNe from solar to a factor of $\sim 10$ above solar, if we allow for the contribution of extra TPs not modeled in detail. Most detailed models produced Ge overabundances up to a factor of $\sim 3$, corresponding to a dilution of 1 part of He-intershell material per roughly 20 parts of envelope material. Hence, we can easily account for the Ge composition of the majority of the observations, in particular, taking into account that substantial observational uncertainties arise from the choice of the level of dust depletion. Even for the mostly Ge-enriched PNe (SwSt 1 and BD +30 3639), for which Ge enhancements have been estimated to be up to a factor of 30 (but could also be as low as $\simeq 5$ if a lower dust depletion is assumed; Sterling \& Dinerstein 2003, 2005), it is conceivable that these overabundances could be reproduced by considering in more detail the effects of stellar model uncertainties, such as the mass loss and treatment of mixing processes that affect the TDU in particular.

The relation between $\mathrm{PNe}$ with $\mathrm{H}$-deficient $\mathrm{CSs}$ and those with the largest Ge abundances could be explained if a late TP during the post-AGB phase (when the envelope mass decreases below $\sim 0.01 M_{\odot}$ ) was responsible for both. These events, de- scribed by Werner \& Herwig (2006), cause the CS to become (partially) H-deficient by mixing and/or burning the small remaining envelope with the larger He intershell, which is enriched in He-burning and $s$-process products. However, such a scenario would also produce larger enhancements of the other $s$-process elements in this type of PNe, while observations show that, on average, the enrichment of $\mathrm{Kr}$ and $\mathrm{Se}$ in $\mathrm{PNe}$ with $\mathrm{H}$-deficient CSs are not significantly different from other PNe (Sterling \& Dinerstein 2006; Sterling 2006). Zhang et al. (2006) recently presented abundances for $\mathrm{Br}, \mathrm{Kr}$, and $\mathrm{Xe}$ derived from $\mathrm{PN}$ observations. For all of these other elements, which we will discuss in detail in a forthcoming paper, results from the Torino code generally produce a good match with the observations (except in the case of $\mathrm{Br}$, which is not observed to be enhanced in $\mathrm{PNe}$ contrary to model predictions), with higher enrichments obtained at lower metallicities, as in the case of Ge discussed here. On the other hand, tentative evidence for the late-TP scenario could come from BD +30 3639, with enhanced Ge and an Fe-deficient CS (Werner \& Herwig 2006), possibly indicating a high level of mixing of $s$-processed material. Further study of the stellar model uncertainties and the extra and/or late-TP scenario together with more accurate observations are needed before anything more conclusive can be said.

A. I. K. warmly thanks Onno Pols at the Astronomical Institute at Utrecht University for his hospitality during a visit while writing this Letter. M. L. gratefully acknowledges the support of the NWO through a VENI grant. R. G. acknowledges support from the Italian MIUR-FIRB project "The Astrophysical Origin of the Heavy Elements beyond Fe." We thank Nick Sterling for helpful discussions and the anonymous referee for a thorough report.

\section{REFERENCES}

Abia, C., \& Isern, J. 1997, MNRAS, 289, L11

Anders, E., \& Grevesse, N. 1989, Geochim. Cosmochim. Acta, 53, 197

Angulo, C., et al. 1999, Nucl. Phys. A, 656, 3

Arlandini, C., Käppeler, F., Wisshak, K., Gallino, R., Lugaro, M., Busso, M., \& Straniero, O. 1999, ApJ, 525, 886

Asplund, M., Grevesse, N., \& Sauval, A. J. 2005, in ASP Conf. Ser. 336, Cosmic Abundances as Records of Stellar Evolution and Nucleosynthesis, ed. T. G. Barnes III \& F. N. Bash (San Francisco: ASP), 25

Busso, M., Gallino, R., Lambert, D. L., Travaglio, C., \& Smith, V. V. 2001, ApJ, 557, 802

Corradi, R. L. M., \& Schwarz, H. E. 1995, A\&A, 293, 871

Cristallo, S., Gallino, R., \& Straniero, O. 2004, Mem. Soc. Astron. Italiana, 75,174

Denissenkov, P. A., \& Tout, C. A. 2003, MNRAS, 340, 722

Dopita, M. A., et al. 1997, ApJ, 474, 188

Gallino, R., Arlandini, C., Busso, M., Lugaro, M., Travaglio, C., Straniero,

O., Chieffi, A., \& Limongi, M. 1998, ApJ, 497, 388

Goriely, S., \& Mowlavi, N. 2000, A\&A, 362, 599

Henry, R. B. C. 1989, MNRAS, 241, 453

Herwig, F. 2000, A\&A, 360, 952

- 2005, ARA\&A, 43, 435

Herwig, F., Langer, N., \& Lugaro, M. 2003, ApJ, 593, 1056

Kaler, J. B. 1978, ApJ, 225, 527

Karakas, A. I., \& Lattanzio, J. C. 2003, Publ. Astron. Soc. Australia, 20, 393

Karakas, A. I., Lugaro, M. A., Wiescher, M., Görres, J., \& Ugalde, C. 2006, ApJ, 643, 471

Langer, N., Heger, A., Wellstein, S., \& Herwig, F. 1999, A\&A, 346, L37

Lugaro, M., Herwig, F., Lattanzio, J. C., Gallino, R., \& Straniero, O. 2003, ApJ, 586, 1305
Lugaro, M., Ugalde, C., Karakas, A. I., Görres, J., Wiescher, M., Lattanzio, J. C., \& Cannon, R. C. 2004, ApJ, 615, 934

Reimers, D. 1975, in Problems in Stellar Atmospheres and Envelopes (New York: Springer), 229

Smith, V. V., Lambert, D. L., \& McWilliam, A. 1987, ApJ, 320, 862

Stanghellini, L., Guerrero, A. M., Cunha, K., Manchado, A., \& Villaver, E. 2006, ApJ, 651, 898

Stanghellini, L., Shaw, R. A., Balick, B., \& Blades, J. C. 2000, ApJ, 534, L167

Sterling, N. C. 2006, Ph.D. thesis, Univ. Texas, Austin

Sterling, N. C., \& Dinerstein, H. L. 2003, RevMexAA, 18, 133

2005, RevMexAA, 23, 1

2006, in IAU Symp. 234, Planetary Nebulae in Our Galaxy and Beyond, ed. M. J. Barlow \& R. H. Méndez (Cambridge: Cambridge Univ. Press), 99

Sterling, N. C., Dinerstein, H. L., \& Bowers, C. W. 2002, ApJ, 578, L55

Straniero, O., Chieffi, A., Limongi, M., Busso, M., Gallino, R., \& Arlandini, C. 1997, ApJ, 478, 332

Straniero, O., Gallino, R., \& Cristallo, S. 2006, Nucl. Phys. A, 777, 311

Thielemann, F.-K., Truran, J. W., \& Arnould, M. 1986, in Advances in Nuclear Astrophysics, ed. E. Vangioni-Flam et al. (Gif-sur-Yvette: Editions Frontières), 525

Vassiliadis, E., \& Wood, P. R. 1993, ApJ, 413, 641

Ventura, P., \& D'Antona, F. 2005, A\&A, 431, 279

Werner, K., \& Herwig, F. 2006, PASP, 118, 183

Zhang, Y., Williams, R., Pellegrini, E., Cavagnolo, K., Baldwin, J. A., Sharpee, B., Phillips, M., \& Liu, X.-W. 2006, in IAU Symp. 234, Planetary Nebulae in Our Galaxy and Beyond, ed. M. J. Barlow \& R. H. Méndez (Cambridge: Cambridge Univ. Press), 549 